\title{
Predictors of Reperfusion in Patients with Acute Ischemic Stroke
}

\author{
A.D. Horsch, J.W. Dankbaar, J.M. Niesten, T. van Seeters, I.C. van der Schaaf, Y. van der Graaf, W.P.Th.M. Mali, and B.K. Velthuis;
} on behalf of the Dutch Acute Stroke Study Investigators

\begin{abstract}
BACKGROUND AND PURPOSE: Ischemic stroke studies emphasize a difference between reperfusion and recanalization, but predictors of reperfusion have not been elucidated. The aim of this study was to evaluate the relationship between reperfusion and recanalization and identify predictors of reperfusion.
\end{abstract}

MATERIALS AND METHODS: From the Dutch Acute Stroke Study, 178 patients were selected with an MCA territory deficit on admission CTP and day 3 follow-up CTP and CTA. Reperfusion was evaluated on CTP, and recanalization on CTA, follow-up imaging. Reperfusion percentages were calculated in patients with and without recanalization. Patient admission and treatment characteristics and admission CT imaging parameters were collected. Their association with complete reperfusion was analyzed by using univariate and multivariate logistic regression.

RESULTS: Sixty percent of patients with complete recanalization showed complete reperfusion (relative risk, 2.60; 95\% Cl, 1.63-4.13). Approximately one-third of patients showed some discrepancy between recanalization and reperfusion status. Lower NIHSS score (OR, 1.06; 95\% Cl, 1.01-1.11), smaller infarct core size (OR, 3.11; 95\% Cl, 1.46-6.66; and OR, 2.40; 95\% Cl, 1.14-5.02), smaller total ischemic area (OR, 4.20; 95\% Cl, 1.91-9.22; and OR, 2.35; 95\% Cl, 1.12-4.91), lower clot burden (OR, 1.35; 95\% Cl, 1.14-1.58), distal thrombus location (OR, 3.02; 95\% $\mathrm{Cl}, 1.76-5.20)$, and good collateral score (OR, 2.84; $95 \% \mathrm{Cl}, 1.34-6.02)$ significantly increased the odds of complete reperfusion. In multivariate analysis, only total ischemic area (OR, 6.12; 95\% Cl, 2.69-13.93; and OR, 1.91; 95\% Cl, 0.91-4.02) was an independent predictor of complete reperfusion.

CONCLUSIONS: Recanalization and reperfusion are strongly associated but not always equivalent in ischemic stroke. A smaller total ischemic area is the only independent predictor of complete reperfusion.

ABBREVIATIONS: DUST = Dutch Acute Stroke Study; RR = relative risk

$\mathbf{P}$ atients with acute ischemic stroke presenting within 4.5 hours are treated with IV-rtPA to dissolve the thrombus and achieve revascularization. ${ }^{1}$ A recent consensus meeting on stroke imaging research (Acute Stroke Imaging Research Roadmap II) suggests that revascularization is a combination of 3 different mechanisms: 1) recanalization, referring to arterial patency; 2) reperfusion,

Received August 11, 2014; accepted after revision December 11.

From the Department of Radiology (A.D.H., J.W.D., J.M.N., T.v.S., I.C.v.d.S., W.P.Th.M.M., B.K.V.), University Medical Center Utrecht, Utrecht, the Netherlands; Department of Radiology (A.D.H.), Rijnstate Hospital, Arnhem, the Netherlands; and Julius Center for Health Sciences and Primary Care (Y.v.d.G.), Utrecht, the Netherlands.

This work was supported by grants from the Netherlands Heart Foundation (2008 T034 and 2012 T061) and the Nuts Ohra Foundation (0903-012).

Please address correspondence to Alexander D. Horsch, MD, MRCS, University Medical Center Utrecht, Heidelberglaan 100, HP E01.132, 3584 CX Utrecht, the Netherlands; e-mail: alexanderhorsch@gmail.com

http://dx.doi.org/10.3174/ajnr.A4283 which refers to antegrade microvascular perfusion; and 3) collateralization, which refers to microvascular perfusion via pial arteries or other anastomotic arterial channels that bypass the primary site of vessel occlusion. ${ }^{2}$ Recanalization, reperfusion, and collateralization can be evaluated by CTA and CTP, which are frequently used in dedicated stroke imaging protocols. An important reason to look at the revascularization mechanisms separately is the concept that recanalization of an arterial occlusion, as visualized on CTA, does not necessarily lead to complete reperfusion and improved clinical outcome. ${ }^{3,4}$ Furthermore, reperfusion can also occur without afferent vessel recanalization through collateralization of the ischemic area by collateral flow. ${ }^{5,6}$

Many previous studies, including those investigating intra-arterial therapy, consider recanalization to be synonymous with reperfusion. ${ }^{7-10}$ Other articles suggest that this assumption is not justified and found reperfusion to be a better predictor of follow-up infarct volume and clinical outcome than recanalization. ${ }^{5,8,9,11-15}$ 
Although recanalization correlates well with improved reperfusion rates, it is unclear which other clinical and imaging factors influence reperfusion. ${ }^{5,6,11,12,16}$ Knowing which factors, available before treatment decisions, predict complete reperfusion could aid in decision-making. Treatment with IV-rtPA, good collateral scores and lesion geography (location of the infarct relative to penumbra), and structure (solitary or multiple infarct areas) have been related to reperfusion status assessed with CT or MR imaging. 5,15,17-19

The aim of this study was to evaluate the relationship between reperfusion and recanalization and to investigate which clinical and CT imaging parameters, available on admission, can help predict complete reperfusion in patients with acute ischemic stroke.

\section{MATERIALS AND METHODS \\ Study Design}

The Dutch Acute Stroke Study (DUST) is a large prospective multicenter cohort study, which aims to assess the additional value of CTP and CTA in predicting the outcome of patients with acute ischemic stroke.

The study protocol has been published previously. ${ }^{20}$ In brief, inclusion criteria for this study were 18 years of age or older, suspected acute ischemic stroke of $<9$ hours' duration, and an NIHSS score of $\geq 2$ (or 1 if an indication for IV-rtPA was present). Exclusion criteria were known renal failure, contraindications to iodinated contrast material, and the presence of another diagnosis on admission NCCT that explained the symptoms. This study was approved by the local institutional ethics review boards of the participating centers. All patients or family gave signed informed consent unless a patient died before consent could be obtained. In that case, the need for consent was waived by the medical ethics committee. ${ }^{20}$

\section{Patient Selection}

From the DUST data base, a consecutive series of patients included between May 2009 and August 2012 was selected from 9 centers. Additional inclusion criteria for this study were the following: 1) perfusion deficit in the MCA territory on admission CTP, and 2) available admission and follow-up CTP and CTA. Exclusion criteria were the following: 1) poor-quality CTP or CTA, 2) absence of 1 of the 2 ASPECTS levels on admission CTP, or 3 ) use of intra-arterial treatment. The inclusion process is clarified in the flow chart (Fig 1).

\section{Predictor Selection}

Clinical variables that were collected included age, sex, history of stroke, admission NIHSS score, IV-rtPA treatment, and time from symptom onset to treatment. Admission imaging variables included the following: infarct core size and total ischemic area from CTP ASPECTS levels and clot burden (clot burden score), thrombus location (ICA, MCA-M1 segment, MCA-M2 segment, and $>$ M2), and collateral score from CTA.

\section{Imaging Protocol}

NCCT, CTP, and CTA of the cervical and cerebral vessel were performed on admission. Multidetector CT scanners were used, with the number of detectors ranging from 40 to 320 (Brilliance 40, Brilliance 64, Brilliance iCT 256; Philips Healthcare, Best, the Netherlands; Sensation 64; Siemens, Erlangen, Ger-

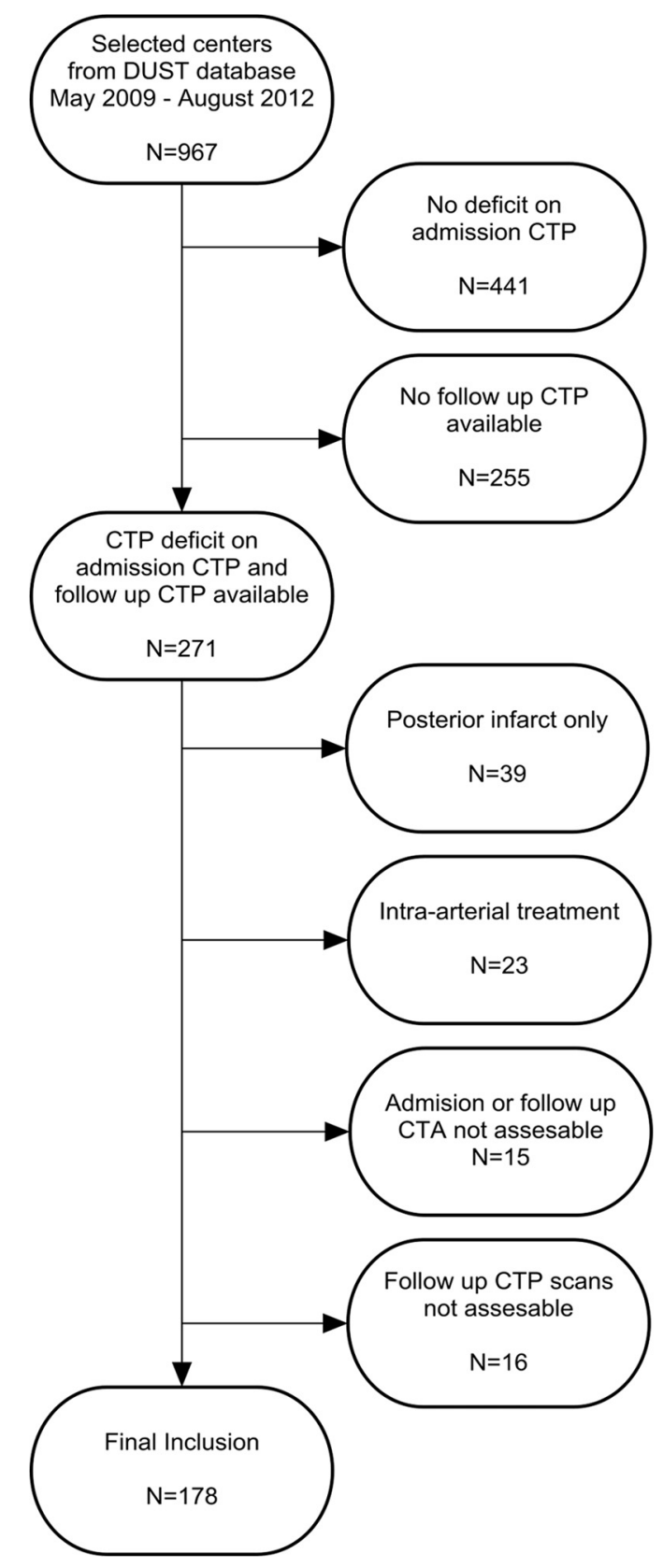

FIG 1. Inclusion flow chart.

many; Aquilion ONE; Toshiba Medical Systems, Tokyo, Japan). NCCT was performed with $120 \mathrm{kV}, 300-375 \mathrm{mAs}$, and a section thickness of $5 \mathrm{~mm} .^{20}$

The CTP, performed with $80 \mathrm{kV}$ and $150 \mathrm{mAs}$, involved successive gantry rotations in cine mode during intravenous administration of $40 \mathrm{~mL}$ of nonionic contrast material followed by 40 $\mathrm{mL}$ of saline with a flow of $6 \mathrm{~mL} / \mathrm{s}^{20} \mathrm{CTP}$ coverage included at least the level of the basal ganglia to the lateral ventricles to be able to assess both ASPECTS levels. ${ }^{21}$

CTA was acquired from the aortic arch to the vertex with $50-70 \mathrm{~mL}$ of contrast followed by $40 \mathrm{~mL}$ of saline, both with a flow of $6 \mathrm{~mL} / \mathrm{s}$. The individual CTA scan delay after intravenous injection was calculated from time-to-peak arterial enhancement on CTP or by trigger-based Hounsfield unit threshold measurement of contrast enhancement in the aortic arch. ${ }^{20}$ 


\section{Imaging Analysis}

CTP. CBF, CBV, MTT, and time-to-peak were automatically calculated from CTP data using commercially available CTP software (Extended Brilliance Workstation 4.5; Philips Healthcare). The nonoccluded ICA or anterior cerebral artery was chosen as the arterial input function. ${ }^{22}$ The superior sagittal sinus was used as the venous output function. The presence of a perfusion deficit on admission was defined as a focal asymmetry on the CBF, CBV, MTT, or time-to-peak map, matching a part or the whole of the MCA flow territory. The total ischemic area was defined with an MTT threshold of $145 \%$ compared with the nonaffected side. Within this area, a CBV value of $<2.0 \mathrm{~mL} / 100 \mathrm{~g}$ was used to define the infarct core. ${ }^{23}$ Infarct core size and total size of the ischemic area were calculated at ASPECTS levels 1 and 2 on admission CTP. The degree of axial CTP coverage was different between scanners because CTP was performed with CT scanners ranging from 40-detector to 320-detector. This range could potentially underestimate the initial ischemic area or the degree of reperfusion. For this reason, we only used the ASPECTS levels to compare patients, to exclude major bias from the difference in the number of detectors.

The presence of reperfusion was analyzed by visual comparison of the admission CTP parameter maps (CBF, CBV, MTT, and time-to-peak) with the follow-up parameter maps. Reperfusion outcome was classified into 2 categories (complete and incomplete reperfusion). Complete reperfusion was defined as the absence of a perfusion deficit on follow-up CTP in the presence of a deficit on admission. All remaining deficits on follow-up CTP, which were not considered to be caused by artifacts, ipsilateral carotid stenosis, or focal hemorrhage, were categorized as incomplete reperfusion. Hyperperfusion was included in the complete reperfusion group, and an enlarged or new perfusion deficit, in the incomplete reperfusion group.

Differences in blood pressure between admission and follow-up CTP have not been taken into account in the assessment of reperfusion status because this assessment was done in a qualitative fashion by visual comparison of the 4 CTP maps and not on image-based quantitative thresholds. It was not expected that potential small differences in the size of the CTP deficit would have changed the category of reperfusion status.

CTA. Admission CTA provided data on clot burden score, collateral score, and intracranial thrombus location. Intracranial thrombus location was divided into 3 groups (intracranial ICA, MCA-M1 segment, and MCA-M2 or more distal occlusion). ${ }^{24-26}$ Thrombus location was classified to the most proximal site of occlusion unless there was a combined extracranial ICA occlusion and a more distal MCA occlusion with an open ICA-top (tandem lesion), which was classified at the level of the MCA occlusion. ${ }^{27}$ Recanalization status was defined qualitatively on follow-up CTA scans as complete or incomplete recanalization. All imaging data were evaluated by 1 of 3 observers (I.C.v.d.S, B.K.V., and J.W.D.), all with at least 5 years of experience in stroke imaging. Only the side of symptoms was provided for the evaluation.

\section{Statistical Analysis}

For all analyses, the complete reperfusion group was compared with the incomplete reperfusion group. Similarly, the complete recanalization group was compared with the incomplete recanalization group. To analyze the relation between complete recanalization and complete reperfusion, absolute and relative risks (RRs) were calculated. Potential determinants of complete reperfusion were tested with univariate and multivariate binary logistic regression analysis, and 95\% confidence intervals were calculated. To reduce the potential influence of non-normal distributions, we recorded the following variables into categories on the basis of tertiles: time to treatment ( $<60$ minutes, $60-120$ minutes, and $\geq 120$ minutes $)$, infarct core size $\left(<300 \mathrm{~mm}^{2}, 300-1400 \mathrm{~mm}^{2}\right.$, and $\left.\geq 1400 \mathrm{~mm}^{2}\right)$, and total ischemic area $\left(<2000 \mathrm{~mm}^{2}, 2000-\right.$ $5000 \mathrm{~mm}^{2}$, and $\geq 5000 \mathrm{~mm}^{2}$ ).

For analysis, patients without visible occlusion on CTA were included in the MCA-M2 or more distal occlusion group. All significant predictors in univariate analysis $(P<.05)$ were used in stepwise backward-elimination multivariate regression analysis. Statistical computations were performed by using SPS 19.0 (IBM, Armonk, New York).

\section{RESULTS}

Inclusion criteria for this study were met in 178 patients (Fig 1). Not all patients in the DUST study received follow-up due to very rapid recovery and discharge before follow-up could be done, poor condition of the patient, impaired renal function, or absence of permission for follow-up. No significant difference in admission NIHSS score was found between patients with or without follow-up CTP and CTA, which suggests that no major preselection occurred.

Baseline clinical and imaging characteristics for the 2 reperfusion groups are summarized in Table 1 . Mean age was 68 years, $39 \%$ were female, median NIHSS score was 9 , and $68 \%$ received IV-rtPA. The infarct core size (479 versus $985 \mathrm{~mm}^{2}$ ) and total ischemic area size (2352 versus $4587 \mathrm{~mm}^{2}$ ) were significantly smaller in the complete reperfusion group compared with the incomplete reperfusion group. In addition, the clot burden was significantly lower in the complete reperfusion group (clot burden score, 9 versus 8 ), and the thrombus was located more distally (M2 or $>\mathrm{M} 2$ in $62 \%$ versus $42 \%$, respectively). A good collateral score was also significantly more frequent in the complete reperfusion group (86\% versus 69\%). No ipsilateral ICA-top occlusion was found on admission in the complete reperfusion group versus $15 \%$ in the incomplete reperfusion group.

Table 2 shows the relation between reperfusion status and recanalization in 152 patients with a visible occlusion and the reperfusion status in 26 patients without a visible occlusion on admission CTA. Complete reperfusion was found in $60 \%$ of patients with complete recanalization and in $23 \%$ in the incomplete-recanalization group (RR, 2.60; 95\% CI, 1.63-4.13). Approximately one-third of patients (32\%) showed some discrepancy between recanalization and reperfusion status. Remarkably, complete reperfusion was found with incomplete recanalization (16 patients), and incomplete reperfusion was found despite complete recanalization (33 patients). Two illustrative cases are shown in Fig 2. In the 26 patients without a 


\begin{tabular}{|c|c|c|c|c|}
\hline & $\begin{array}{l}\text { All Patients } \\
(n=178)\end{array}$ & $\begin{array}{l}\text { Incomplete Reperfusion } \\
\qquad(n=92,52 \%)\end{array}$ & $\begin{array}{l}\text { Complete Reperfusion } \\
\text { ( } n=86,48 \%)\end{array}$ & $P$ Value \\
\hline \multicolumn{5}{|l|}{ Clinical parameters } \\
\hline Age (yr) (mean) (SD) & $68(13)$ & $68(14)$ & $67(13)$ & .92 \\
\hline Female sex (No.) (\%) & 69 (39) & $34(37)$ & $35(41)$ & .61 \\
\hline Prior stroke (No.) (\%) & $35(20)$ & $15(16)$ & $20(23)$ & .24 \\
\hline NIHSS score (median) (IQR) & $9(5-15)$ & $12(5-16)$ & $8(4-13)$ & .06 \\
\hline \multicolumn{5}{|l|}{ Treatment } \\
\hline rtPA (No.) (\%) & $121(68)$ & $61(66)$ & $60(70)$ & .62 \\
\hline Time to treatment ${ }^{a}(\mathrm{~min})$ (median) (IQR) & $100(73-130)$ & $105(75-127)$ & 99 (70-151) & .89 \\
\hline \multicolumn{5}{|l|}{ Imaging parameters } \\
\hline Time to admission scan (min) (median) (IQR) & $105(66-170)$ & $105(71-157)$ & $105(61-187)$ & .62 \\
\hline Time to follow-up (day) (mean) (SD) & $3.2(1.3)$ & $3.3(1.3)$ & $3.1(1.2)$ & .19 \\
\hline \multicolumn{5}{|l|}{ CTP } \\
\hline Size of infarct core ${ }^{b}\left(\mathrm{~mm}^{2}\right)$ (median) (IQR) & $630(187-1758)$ & $985(293-2659)$ & 479 (115-1332) & $.002^{c}$ \\
\hline Size of total ischemic area $\left(\mathrm{mm}^{2}\right)$ (median) (IQR) & $3629(1551-5605)$ & $4587(2848-6248)$ & $2352(734-4328)$ & $.0005^{\mathrm{c}}$ \\
\hline \multicolumn{5}{|l|}{ CTA } \\
\hline Clot burden score (median) (IQR) & $8.0(6.0-9.0)$ & $8.0(6.0-9.0)$ & $9.0(7.0-9.0)$ & $.003^{c}$ \\
\hline Thrombus location, ${ }^{\mathrm{d}}$ ICA (No.) (\%) & $13(9)$ & $13(15)$ & 0 & $.01^{\mathrm{c}}$ \\
\hline Thrombus location, ${ }^{d} \mathrm{Ml}$ (No.) (\%) & $62(41)$ & $37(43)$ & $25(38)$ & $.01^{\mathrm{c}}$ \\
\hline Thrombus location, ${ }^{d}$ M2 or >M2 (No.) (\%) & $77(51)$ & $36(42)$ & $41(62)$ & $.01^{\mathrm{c}}$ \\
\hline Good collateral score (No.) (\%) & $137(77)$ & $63(69)$ & $74(86)$ & $.005^{c}$ \\
\hline
\end{tabular}

Note:-IQR indicates interquartile range.

a Only 121 patients received IV-rtPA.

${ }^{\mathrm{b}}$ Size of infarct core and total ischemic area at both ASPECTS levels combined.

c Significant.

d Only 152 cases because 26 patients had no visible occlusion on admission CTA.

e All patients without visible artery occlusion on admission CTA had a good collateral score.

Table 2: Outcome summary-relation between reperfusion and recanalization $^{\text {a }}$

\begin{tabular}{lcrr}
\hline & $\begin{array}{c}\text { Incomplete } \\
\text { Reperfusion }\end{array}$ & $\begin{array}{c}\text { Complete } \\
\text { Reperfusion }\end{array}$ & Total \\
\hline $\begin{array}{l}\text { Reperfusion and recanalization } \\
(n=152)\end{array}$ & & & \\
Incomplete recanalization & $53(77)$ & $16(23)$ & $69(100)$ \\
$\quad$ Complete recanalization & $33(40)$ & $50(60)$ & $83(100)$ \\
$\quad$ Total & $86(57)$ & $66(43)$ & $152(100)$ \\
Reperfusion without visible & & & \\
$\quad$ occlusion on admission & & & \\
$\quad$ CTA ( $n=26)$ & & & \\
$\quad$ No occlusion on admission & $6(23)$ & $20(77)$ & $26(100)$ \\
\hline a Data are No. (\% in rows). & & &
\end{tabular}

visible occlusion on admission CTA, most patients (77\%) showed complete reperfusion.

The univariate logistic regression analysis is shown in Table 3. Lower NIHSS score (OR, 1.06; 95\% CI, 1.01-1.11), smaller infarct core size (OR, 3.11; 95\% CI, 1.46-6.66; and OR, 2.40; 95\% CI, 1.14-5.02), smaller total ischemic area (OR, 4.20; 95\% CI, 1.919.22; and OR, 2.35; 95\% CI, 1.12-4.91), lower clot burden (OR, 1.35; 95\% CI, 1.14-1.58), distal thrombus location (OR, 3.02; $95 \% \mathrm{CI}, 1.76-5.20$ ), and good collateral score (OR, 2.84; 95\% CI, 1.34-6.02) significantly increased the odds of complete reperfusion. Age, sex, or IV-rtPA treatment did not influence complete reperfusion. In multivariate analysis, a smaller total ischemic area (OR, 6.12; 95\% CI, 2.69-13.93; and OR, 1.91; 95\% CI, 0.91-4.02) was the only independent predictor of complete reperfusion (not shown in the Tables).

\section{DISCUSSION}

This study shows that although reperfusion is strongly related to recanalization in acute ischemic stroke, reperfusion and recanalization do not always occur in unison. An important finding is that none of the patients with an ipsilateral intracranial ICA occlusion showed complete reperfusion. Factors that help predict complete reperfusion are lower NIHSS score on admission, lower clot burden, more distal thrombus location and a good collateral score (on CTA), and smaller infarct core and smaller total ischemic area (on CTP). In multivariate analysis, only total ischemic area was an independent predictor of complete reperfusion.

\section{Recanalization versus Reperfusion}

The proportion of complete recanalization and complete reperfusion in our study compares with values described in the literature, $22 \%-60 \%$ for recanalization and $26 \%-79 \%$ for reperfusion. ${ }^{15-18}$ Our data confirm the suggestion that recanalization and reperfusion are closely related but not interchangeable. The discrepancy of incomplete reperfusion in the presence of complete recanalization can be caused by either the break-up of the primary clot into fragments leading to distal embolization of smaller vasculature not visible on CTA, reversible incomplete microcirculatory reperfusion, or the irreversible no-reflow phenomenon. ${ }^{3,28-30}$ Reperfusion without recanalization of the proximal occlusion is thought to be mediated by collateralization (through pial arteries or other anastomotic channels). ${ }^{2}$ Patients without an identifiable occlusion on admission CTA had higher complete reperfusion rates in comparison with patients with a visible occlusion. Undetected microthrombi in distal vessels may account for this higher rate because they are more likely to dissolve.

\section{Predictors of Reperfusion}

Thrombus location was a predictor of complete reperfusion in our study. Distal MCA occlusions showed better reperfusion rates on follow-up compared with more proximal MCA or ICA-top occlusions. A positive relation between distal thrombus location 


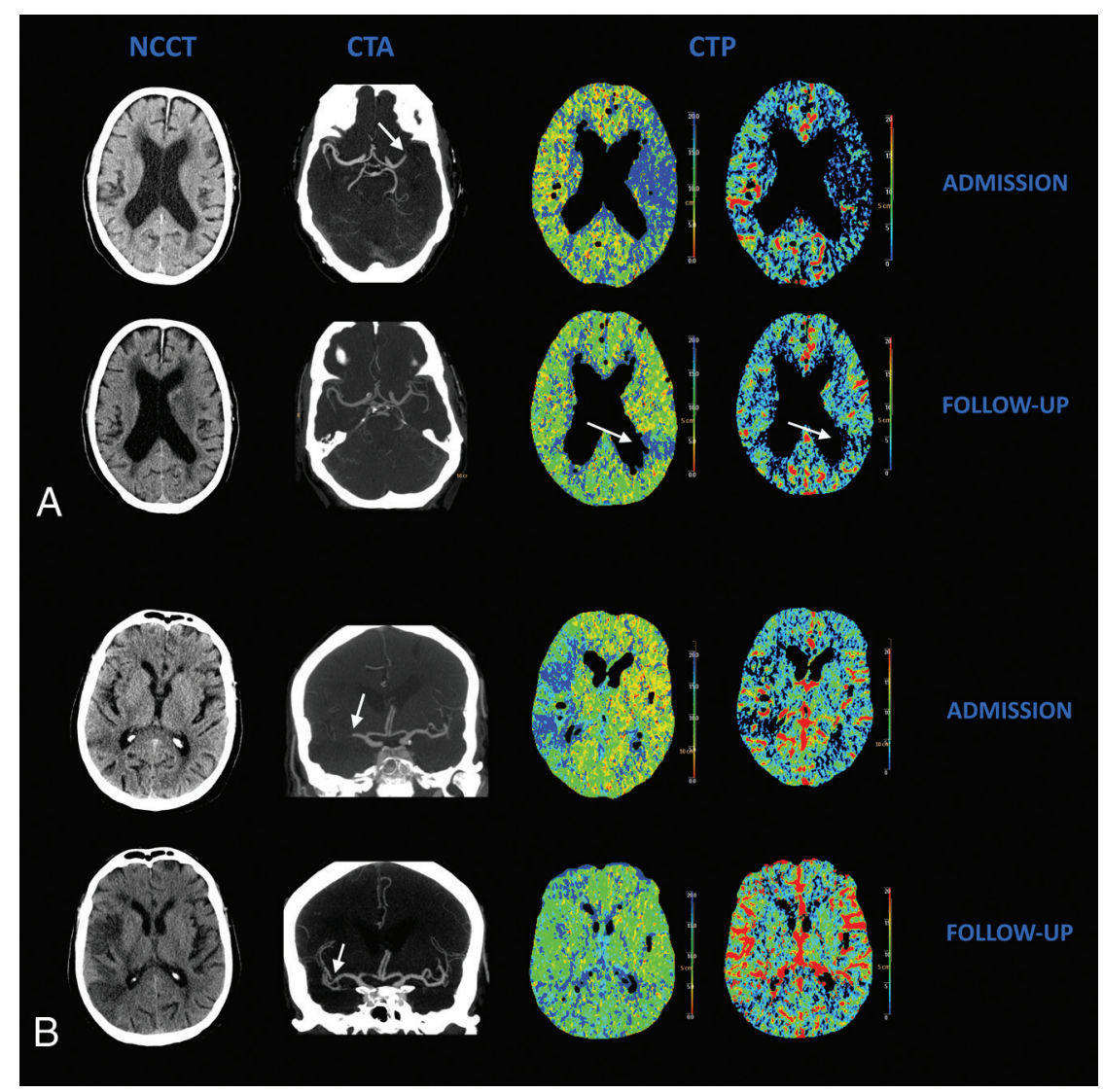

FIG 2. Relation between recanalization and reperfusion. A, A patient with complete recanalization without complete reperfusion. Admission NCCT shows no early CT signs. Follow-up NCCT shows infarction of the basal ganglia. Admission CTA shows occlusion of the Ml segment (arrow). Follow-up CTA shows complete recanalization; no distal M3 occlusion could be found. Admission CTP shows a large area of decreased MTT and CBV in ASPECTS M5 and M6. Follow-up CTP shows a residual perfusion deficit ASPECTS M6 on the MTT and CBV maps (arrow). B, A patient with incomplete recanalization but complete reperfusion. Admission NCCT shows some early CT signs in the MCA territory. Follow-up NCCT shows areas of infarction in a large part of the MCA territory. Admission CTA shows an occlusion in the M1 and M2 segments of the MCA. Follow-up CTA shows a short residual occlusion in an M2 segment (arrows). Admission CTP shows a large area of decreased MTT and CBV in ASPECTS M1-M3, which has completely resolved on follow-up.

Table 3: Predictors of complete reperfusion $(N=178)$

\begin{tabular}{|c|c|c|c|}
\hline & OR & $95 \% \mathrm{Cl}$ & $P$ Value \\
\hline \multicolumn{4}{|l|}{ Clinical parameters } \\
\hline Age (yr) & 1.00 & $0.98-1.02$ & .86 \\
\hline Female sex & 1.17 & $0.64-2.14$ & .61 \\
\hline NIHSS score & 1.06 & 1.01-1.11 & $.03^{\mathrm{a}}$ \\
\hline rtPA & 1.17 & $0.62-2.21$ & .62 \\
\hline Time to treatment, ${ }^{b} 60-120$ min versus $<60 \mathrm{~min}$ & 0.54 & $0.17-1.69$ & .29 \\
\hline Time to treatment, ${ }^{b} \geq 120$ min versus $<60 \mathrm{~min}$ & 0.78 & $0.23-2.61$ & .68 \\
\hline \multicolumn{4}{|l|}{ Imaging parameters } \\
\hline Size infarct core, ${ }^{c}<300$ versus $\geq 1400 \mathrm{~mm}^{2}$ & 3.11 & $1.46-6.66$ & $.003^{\mathrm{a}}$ \\
\hline Size infarct core, ${ }^{c} 300-1400$ versus $\geq 1400 \mathrm{~mm}^{2}$ & 2.40 & $1.14-5.02$ & $.02^{\mathrm{a}}$ \\
\hline Size total ischemic area, ${ }^{c}<2000$ vs $\geq 5000$ & 4.20 & $1.91-9.22$ & $.004^{\mathrm{a}}$ \\
\hline Size total ischemic area, ${ }^{c} 2000-5000$ versus $\geq 5000 \mathrm{~mm}^{2}$ & 2.35 & $1.12-4.91$ & $.02^{\mathrm{a}}$ \\
\hline Clot burden score & 1.35 & $1.14-1.58$ & $.001^{\mathrm{a}}$ \\
\hline Thrombus location, ICA, $\mathrm{M} 1$ or $\mathrm{M} 2$, and $>\mathrm{M} 2$ & 3.02 & $1.76-5.20$ & $.006^{\mathrm{a}}$ \\
\hline Good collateral score ${ }^{\mathrm{d}}$ & 2.84 & $1.34-6.02$ & $.01^{\mathrm{a}}$ \\
\hline
\end{tabular}

a $P<.05$ is significant.

${ }^{b}$ Only 121 patients received IV-rtPA.

'Size of infarct core and total ischemic area at both ASPECTS levels combined.

${ }^{\mathrm{d}}$ All patients without visible artery occlusion on admission CTA had a good collateral score.

and recanalization has been found in multiple studies. ${ }^{7,31-33}$ In contrast, Lemmens et $\mathrm{al}^{34}$ found no relation between distal occlusion and recanalization, though they did find a better clinical response with increased reperfusion. Moreover, none of our patients with an intracranial carotid occlusion showed complete reperfusion (despite complete recanalization in $15 \%$ and $54 \%$ receiving IV-rtPA in this patient group of 13 patients). These findings suggest that those specific patients may need intraarterial thrombolysis or mechanical thrombectomy. A recent review showed that stent placement and mechanical thrombectomy in patients with an intracranial ICA occlusion are associated with higher recanalization rates and better functional outcome. ${ }^{35}$

Few articles describe predictors of brain tissue reperfusion. The most frequently found imaging predictor of reperfusion was recanalization in both CT and MR imaging studies. 5,6,11,16,17,36 However, recanalization is not a variable that can be assessed before treatment. Good collateral scores, treatment with alteplase or tenecteplase, older age, and lesion geography and structure were also associated with better reperfusion. 5,15,17-19,37

Only total ischemic area was an independent predictor of complete reperfusion in our study. This is not surprising because a smaller size of the total ischemic area is the result of a more distal thrombus location and good collateral status. The effect of these variables is therefore most likely represented by the total ischemic area in our multivariate analysis.

It is unclear why we could not demonstrate a significant relation between IV-rtPA treatment and complete reperfusion. Christoforidis et $\mathrm{al}^{38}$ suggested no benefit from thrombolysis in patients with poor collaterals and a more distal occlusion site, but in our population, we did not find a worse reperfusion rate in the patient group treated with IV-rtPA. Another reason could be a difference in population characteristics between treated and nontreated patients, with a higher admission NIHSS score in the IVrtPA-treated patients.

Although some predictors of reperfusion (size of the infarct core, size of the total ischemic area) have not been related to recanalization, most reperfusion predictors (NIHSS score, clot burden score, thrombus location, collateral score) have been established for recanalization..$^{7,16,24,32,39-45}$ In addition, several articles describing predictors of reperfusion or recanalization showed a good correlation between these predic- 
tive factors and outcome (final infarct size and $\mathrm{mRS}$ at 3 months). ${ }^{6,11,16}$ This is not surprising because reperfusion is a parameter situated between recanalization and outcome. Our data confirm that these relations are already established at day 3, relatively early in the clinical course. This finding suggests that follow-up reperfusion data could be used as a surrogate end point.

There are some limitations to this study. First, our patient group is seemingly different from those in many previous stroke articles because of a shorter time to scan, lower NIHSS scores, and higher IV-rtPA treatment rate. However, with improved stroke awareness and faster stroke protocols, our population is probably more representative of the current stroke populations.

Second, the time to follow-up was approximately 3 days. Most brain cells die early in ischemic conditions, but recanalization is known to continue even after the focal areas have infarcted. Timely and clinically meaningful recanalization and reperfusion may be better demonstrated when follow-up is performed at an earlier time point.

Third, assessment of both recanalization and reperfusion was not done quantitatively but in a 2-point scale, a method also previously used. ${ }^{46-48}$ The predictive value can improve with more quantitative assessment, but small lesions are prone to measurement errors. ${ }^{15}$ Moreover, qualitative assessment is easier and shows good agreement in clinical practice, especially with limited lesion coverage. ${ }^{49}$

Fourth, the 26 patients with a peripheral perfusion deficit, who did not show an occlusion on CTA, were included in the M2$\mathrm{MCA} /$ distal occlusion group. It was assumed that the peripheral perfusion deficit in these patients was caused by a distal occlusion, not detectable on CTA. Possibly, some of these distal occlusions may have resulted from fragmentation of a larger thrombus and thus (partial) recanalization before the initial imaging, However, this cause seems unlikely because the median time to treatment in our study was only 100 minutes, which leaves little time for early recanalization to occur.

\section{CONCLUSIONS}

This study confirms that recanalization and reperfusion outcomes in ischemic stroke are significantly related but are not always interchangeable. Lower NIHSS scores, smaller size of the infarct core, smaller total ischemic area, lower clot burden, more distal intracranial thrombus location, and good collateral scores have been identified as predictors of complete reperfusion. A smaller total ischemic area is the only independent predictor of complete reperfusion.

\section{APPENDIX}

The following are the DUST investigators: Academic Medical Center, Amsterdam, the Netherlands (C.B. Majoie, Y.B. Roos); Catharina Hospital, Eindhoven, the Netherlands (L.E. Duijm, K. Keizer); Erasmus Medical Center, Rotterdam, the Netherlands (A. van der Lugt, D.W. Dippel); Gelre Hospitals, Apeldoorn, the Netherlands (K.E. Droogh-de Greve, H.P. Bienfait); Leiden University Medical Center, Leiden, the Netherlands (M.A. van Walderveen, M.J. Wermer); Medical Center Haaglanden, The Hague, the Netherlands (G.J. Lycklama à Nijeholt, J. Boiten); Onze Lieve Vrouwe Gasthuis, Amsterdam, the Netherlands (D. Duyndam,
V.I. Kwa); Radboud University Nijmegen Medical Center, Nijmegen, the Netherlands (F.J. Meijer, E.J. van Dijk); Rijnstate Hospital, Arnhem, the Netherlands (F.O. Kesselring, J. Hofmeijer); St. Antonius Hospital, Nieuwegein, the Netherlands (J.A. Vos, W.J. Schonewille); St. Elisabeth Hospital, Tilburg, the Netherlands (W.J. van Rooij, P.L. de Kort); St. Franciscus Gasthuis, Rotterdam, the Netherlands (C.C. Pleiter, S.L. Bakker); VU Medical Center, Amsterdam, the Netherlands (J. Bot, M.C. Visser); University Medical Center Utrecht, Utrecht, the Netherlands (B.K. Velthuis, I.C. van der Schaaf, J.W. Dankbaar, W.P. Mali, T. van Seeters, A.D. Horsch, J.M. Niesten, G.J. Biessels, L.J. Kappelle, M.J. Luitse, Y. van der Graaf).

Disclosures: Jan W. Dankbaar-RELATED: Grant: Netherlands Heart Foundation (No. 2012T61). Birgitta K. Velthuis—RELATED: Grant: Netherlands Heart Foundation, * Nuts Ohra Foundation, ${ }^{*}$ Comments: This study was supported by grants from the Netherlands Heart Foundation (2008 T034 and 2012 T061) and the Nuts Ohra Foundation (0903-012).

\section{REFERENCES}

1. Hacke W, Donnan G, Fieschi C, et al. Association of outcome with early stroke treatment: pooled analysis of ATLANTIS, ECASS, and NINDS rt-PA stroke trials. Lancet 2004;363:768-74

2. Wintermark M, Albers GW, Broderick JP, et al; for the Stroke Imaging Research (STIR) and Virtual International Stroke Trials Archive (VISTA)-Imaging Investigators. Acute Stroke Imaging Research Roadmap II. Stroke 2013;44:2628-39

3. Dalkara T, Arsava EM. Can restoring incomplete microcirculatory reperfusion improve stroke outcome after thrombolysis? J Cereb Blood Flow Metab 2012;32:2091-99

4. Zaidat OO, Yoo AJ, Khatri P, et al. Recommendations on angiographic revascularization grading standards for acute ischemic stroke: a consensus statement. Stroke 2013;44:2650-63

5. Soares BP, Tong E, Hom J, et al. Reperfusion is a more accurate predictor of follow-up infarct volume than recanalization: a proof of concept using CT in acute ischemic stroke patients. Stroke 2010;41:e34-40

6. Albers GW, Thijs VN, Wechsler L, et al. Magnetic resonance imaging profiles predict clinical response to early reperfusion: the diffusion and perfusion imaging evaluation for understanding stroke evolution (DEFUSE) study. Ann Neurol 2006;60:508-17

7. Murphy A, Symons SP, Hopyan J, et al. Factors influencing clinically meaningful recanalization after IV-rtPA in acute ischemic stroke. AJNR Am J Neuroradiol 2013;34:146-52

8. Furlan AJ, Eyding D, Albers GW, et al. Dose Escalation of Desmoteplase for Acute Ischemic Stroke (DEDAS): evidence of safety and efficacy 3 to 9 hours after stroke onset. Stroke 2006;37:1227-31

9. Hacke W, Albers G, Al-Rawi Y, et al. The Desmoteplase in Acute Ischemic Stroke Trial (DIAS): a phase II MRI-based 9-hour window acute stroke thrombolysis trial with intravenous desmoteplase. Stroke 2005;36:66-73

10. Fiehler J, Remmele C, Kucinski T, et al. Reperfusion after severe local perfusion deficit precedes hemorrhagic transformation: an MRI study in acute stroke patients. Cerebrovasc Dis 2005;19:117-24

11. Eilaghi A, Brooks J, d'Esterre C, et al. Reperfusion is a stronger predictor of good clinical outcome than recanalization in ischemic stroke. Radiology 2013;269:240-48

12. De Silva DA, Fink JN, Christensen S, et al. Assessing reperfusion and recanalization as markers of clinical outcomes after intravenous thrombolysis in the Echoplanar Imaging Thrombolytic Evaluation Trial (EPITHET). Stroke 2009;40:2872-74

13. Yoo AJ, Chandra RV, Lev MH. Revascularization grading: the devil is in the details. Radiology 2013;269:6-7

14. Soares BP, Chien JD, Wintermark M. MR and CT monitoring of recanalization, reperfusion, and penumbra salvage: everything that recanalizes does not necessarily reperfuse! Stroke 2009;40:S24-27 
15. Davis SM, Donnan GA, Parsons MW, et al. Effects of alteplase beyond $3 \mathrm{~h}$ after stroke in the Echoplanar Imaging Thrombolytic Evaluation Trial (EPITHET): a placebo-controlled randomised trial. Lancet Neurol 2008;7:299-309

16. Derex L, Nighoghossian N, Hermier M, et al. Influence of pretreatment MRI parameters on clinical outcome, recanalization and infarct size in 49 stroke patients treated by intravenous tissue plasminogen activator. J Neurol Sci 2004;225:3-9

17. Parsons M, Spratt N, Bivard A, et al. A randomized trial of tenecteplase versus alteplase for acute ischemic stroke. $N$ Engl J Med 2012;366:1099-107

18. Ogata T, Christensen S, Nagakane Y, et al. The effects of alteplase 3 to 6 hours after stroke in the EPITHET-DEFUSE combined dataset: post hoc case-control study. Stroke 2013;44:87-93

19. Olivot JM, Mlynash M, Thijs VN, et al. Geography, structure, and evolution of diffusion and perfusion lesions in Diffusion and perfusion imaging Evaluation For Understanding Stroke Evolution (DEFUSE). Stroke 2009;40:3245-51

20. van Seeters T, Biessels GJ, van der Schaaf IC, et al. Prediction of outcome in patients with suspected acute ischaemic stroke with CT perfusion and CT angiography: the Dutch Acute Stroke Trial (DUST) study protocol. BMC Neurol 2014;14:37

21. Barber PA, Demchuk AM, Zhang J, et al. Validity and reliability of a quantitative computed tomography score in predicting outcome of hyperacute stroke before thrombolytic therapy. Lancet 2000;355: $1670-74$

22. Niesten JM, van der Schaaf IC, Riordan AJ, et al. Optimisation of vascular input and output functions in CT-perfusion imaging using 256 (or more)-slice multidetector CT. Eur Radiol 2013;23:1242-49

23. Wintermark M, Flanders AE, Velthuis B, et al. Perfusion-CT assessment of infarct core and penumbra: receiver operating characteristic curve analysis in $\mathbf{1 3 0}$ patients suspected of acute hemispheric stroke. Stroke 2006;37:979-85

24. Tan IYL, Demchuk AM, Hopyan J, et al. CT angiography clot burden score and collateral score: correlation with clinical and radiologic outcomes in acute middle cerebral artery infarct. AJNR Am J Neuroradiol 2009;30:525-31

25. Tan JC, Dillon WP, Liu S, et al. Systematic comparison of perfusion-CT and CT-angiography in acute stroke patients. Ann Neurol 2007;61:533-43

26. Puetz V, Dzialowski I, Hill MD, et al. Intracranial thrombus extent predicts clinical outcome, final infarct size and hemorrhagic transformation in ischemic stroke: the clot burden score. Int J Stroke 2008;3:230-36

27. El-Mitwalli A, Saad M, Christou I, et al. Clinical and sonographic patterns of tandem internal carotid artery/middle cerebral artery occlusion in tissue plasminogen activator-treated patients. Stroke 2002;33:99-102

28. Molina CA, Alvarez-Sabin J. Recanalization and reperfusion therapies for acute ischemic stroke. Cerebrovasc Dis 2009;27(suppl 1): 162-67

29. del Zoppo GJ. Virchow's triad: the vascular basis of cerebral injury. Rev Neurol Dis 2008;5(suppl 1):S12-21

30. Wong $\mathrm{CH}$, Crack PJ. Modulation of neuro-inflammation and vascular response by oxidative stress following cerebral ischemia-reperfusion injury. Curr Med Chem 2008;15:1-14

31. Mendonça N, Rodriguez-Luna D, Rubiera M, et al. Predictors of tissue-type plasminogen activator nonresponders according to location of vessel occlusion. Stroke 2012;43:417-21
32. Bhatia R, Hill MD, Shobha N, et al. Low rates of acute recanalization with intravenous recombinant tissue plasminogen activator in ischemic stroke: real-world experience and a call for action. Stroke 2010;41:2254-58

33. Yeo LL, Paliwal P, Teoh HL, et al. Timing of recanalization after intravenous thrombolysis and functional outcomes after acute ischemic stroke. JAMA Neurol 2013;70:353-58

34. Lemmens R, Mlynash M, Straka M, et al. Comparison of the response to endovascular reperfusion in relation to site of arterial occlusion. Neurology 2013;81:614-18

35. Kappelhof M, Marquering HA, Berkhemer OA, et al. Intra-arterial treatment of patients with acute ischemic stroke and internal carotid artery occlusion: a literature review. J Neurointerv Surg 2015;7:8-15

36. Marks MP, Olivot JM, Kemp S, et al. Patients with acute stroke treated with intravenous tPA 3-6 hours after stroke onset: correlations between MR angiography findings and perfusion- and diffusion-weighted imaging in the DEFUSE study. Radiology 2008; 249:614-23

37. Lansberg MG, Thijs VN, Bammer R, et al. The MRA-DWI mismatch identifies patients with stroke who are likely to benefit from reperfusion. Stroke 2008;39:2491-96

38. Christoforidis GA, Mohammad Y, Kehagias D, et al. Angiographic assessment of pial collaterals as a prognostic indicator following intra-arterial thrombolysis for acute ischemic stroke. AJNR Am J Neuroradiol 2005;26:1789-97

39. Shhadeh A, Garg A, Hassan AE, et al. Recanalization following various endovascular modalities for treatment of anterior circulation acute ischemic strokes. J Vasc Interv Neurol 2012;5:10-16

40. Tandberg Askevold E, Naess H, Thomassen L. Predictors for recanalization after intravenous thrombolysis in acute ischemic stroke. $J$ Stroke Cerebrovasc Dis 2007;16:21-24

41. Saqqur M, Uchino K, Demchuk AM, et al. Site of arterial occlusion identified by transcranial Doppler predicts the response to intravenous thrombolysis for stroke. Stroke 2007;38:948-54

42. Legrand L, Naggara O, Turc G, et al. Clot burden score on admission T2*-MRI predicts recanalization in acute stroke. Stroke 2013;44: $1878-84$

43. Nam HS, Kim EY, Kim SH, et al. Prediction of thrombus resolution after intravenous thrombolysis assessed by CT-based thrombus imaging. Thromb Haemost 2012;107:786-94

44. Zangerle A, Kiechl S, Spiegel M, et al. Recanalization after thrombolysis in stroke patients: predictors and prognostic implications. Neurology 2007;68:39-44

45. Nambiar V, Sohn SI, Almekhlafi MA, et al. CTA collateral status and response to recanalization in patients with acute ischemic stroke. AJNR Am J Neuroradiol 2014;35:884-90

46. Bivard A, Spratt N, Levi C, et al. Perfusion computer tomography: imaging and clinical validation in acute ischaemic stroke. Brain 2011;134:3408-16

47. Kloska SP, Dittrich R, Fischer T, et al. Perfusion CT in acute stroke: prediction of vessel recanalization and clinical outcome in intravenous thrombolytic therapy. Eur Radiol 2007;17:2491-98

48. Olivot JM, Mlynash M, Thijs VN, et al. Relationships between cerebral perfusion and reversibility of acute diffusion lesions in DEFUSE: insights from RADAR. Stroke 2009;40:1692-97

49. Luby M, Ku KD, Latour LL, et al. Visual perfusion-diffusion mismatch is equivalent to quantitative mismatch. Stroke 2011;42: $1010-14$ 